ORIGINAL ARTICLE

\title{
Linking a dermal permeation and an inhalation model to a simple pharmacokinetic model to study airborne exposure to di(n-butyl) phthalate
}

Matthew Lorber ${ }^{1}$, Charles J. Weschler ${ }^{2,3}$, Glenn Morrison ${ }^{4}$, Gabriel Bekö ${ }^{3}$, Mengyan Gong ${ }^{5}$, Holger M. Koch ${ }^{6}$, Tunga Salthammer ${ }^{7}$, Tobias Schripp ${ }^{7}$, Jørn Toftum ${ }^{3}$ and Geo Clausen ${ }^{3}$

Six males clad only in shorts were exposed to high levels of airborne di(n-butyl) phthalate (DnBP) and diethyl phthalate (DEP) in chamber experiments conducted in 2014. In two $6 \mathrm{~h}$ sessions, the subjects were exposed only dermally while breathing clean air from a hood, and both dermally and via inhalation when exposed without a hood. Full urine samples were taken before, during, and for $48 \mathrm{~h}$ after leaving the chamber and measured for key DnBP and DEP metabolites. The data clearly demonstrated high levels of DnBP and DEP metabolite excretions while in the chamber and during the first $24 \mathrm{~h}$ once leaving the chamber under both conditions. The data for DnBP were used in a modeling exercise linking dose models for inhalation and transdermal permeation with a simple pharmacokinetic model that predicted timing and mass of metabolite excretions. These models were developed and calibrated independent of these experiments. Tests included modeling of the "hood-on" (transdermal penetration only), "hood-off" (both inhalation and transdermal) scenarios, and a derived "inhalation-only" scenario. Results showed that the linked model tended to duplicate the pattern of excretion with regard to timing of peaks, decline of concentrations over time, and the ratio of DnBP metabolites. However, the transdermal model tended to overpredict penetration of DnBP such that predictions of metabolite excretions were between 1.1 and 4.5 times higher than the cumulative excretion of DnBP metabolites over the $54 \mathrm{~h}$ of the simulation. A similar overprediction was not seen for the "inhalation-only" simulations. Possible explanations and model refinements for these overpredictions are discussed. In a demonstration of the linked model designed to characterize general population exposures to typical airborne indoor concentrations of DnBP in the United States, it was estimated that up to one-quarter of total exposures could be due to inhalation and dermal uptake.

Journal of Exposure Science and Environmental Epidemiology (2017) 27, 601-609; doi:10.1038/jes.2016.48; published online 17 August 2016

Keywords: dermal permeation modeling; DnBP; pharmacokinetic modeling

\section{INTRODUCTION}

Diethyl phthalate (DEP) and di(n-butyl) phthalate (DnBP) are among the most abundant man-made compounds found in indoor air. $^{1-3}$ Furthermore, the metabolites of phthalate esters, including these two, are often the most abundant metabolites of man-made compounds found in human urine. ${ }^{4}$ Humans are exposed to these compounds in their diets via inhalation, dust ingestion, and dermal absorption..$^{5-7}$ Clark et al. ${ }^{8}$ have shown that pathways other than diet can be important for these two phthalates, and in particular, consumer products may dominate in certain circumstances for DEP. Dust ingestion may be important for DnBP, but it is anticipated to be much less important for DEP. DnBP is typically present in household dust at a mass fraction of 5 to $100 \mu \mathrm{g} / \mathrm{g}$, whereas DEP, with a higher vapor pressure, has a typical mass fraction of 1 to $10 \mu \mathrm{g} / \mathrm{g}$ (see Table 3 in Langer et al. ${ }^{9}$ ). Given the elevated concentrations of DEP and DnBP in indoor air, inhalation could be an important route of exposure. A recent study with human volunteers under controlled conditions has demonstrated that, following a $6 \mathrm{~h}$ exposure, dermal uptake from air is comparable to inhalation intake. ${ }^{10}$

Dermal uptake of small organic molecules, directly from air, has been amply documented. ${ }^{11-13}$ However, only in the past several years has uptake from air been considered a potentially significant pathway for certain higher molecular weight organic compounds such as phthalate esters. ${ }^{14}$ Weschler and Nazaroff ${ }^{15}$ developed a steady-state model to estimate the "overall dermal permeability coefficient" (i.e., from air through skin to blood) for organic compounds based on their fundamental physical-chemical properties. Their model was based on a mechanistic model developed by Mitragotri ${ }^{16}$ to predict transdermal permeability of organic species from aqueous solution. Gong et al. ${ }^{17}$ extended these ideas and crafted a transient model to estimate the transport of a gas-phase organic compound from air through skin to blood under dynamic conditions. Morrison et al. ${ }^{18}$ modified

\footnotetext{
${ }^{1}$ Office of Research and Development, US EPA, Washington, District of Columbia, USA; ${ }^{2}$ Environmental and Occupational Health Sciences Institute, Rutgers University, Piscataway, New Jersey, USA; ${ }^{3}$ International Centre for Indoor Environment and Energy, Department of Civil Engineering, Technical University of Denmark, Lyngby, Denmark; ${ }^{4}$ Civil, Architectural and Environmental Engineering, Missouri University of Science and Technology, Rolla, Missouri, USA; ${ }^{5}$ National Institute of Standards and Technology, Gaithersburg, Maryland, USA; ${ }^{6}$ Institute for Prevention and Occupational Medicine of the German Social Accident Insurance, Institute of the Ruhr-Universität Bochum (IPA), Bochum, Germany

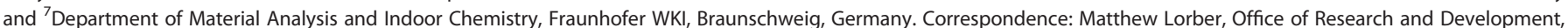
US EPA, NCEA (8623N), 1200 Pennsylvania Avenue, NW, Washington, DC 20460, USA.
}

Tel.: 703 347-8535. Fax: 703 347-8690.

E-mail: lorber.matthew@epa.gov

Received 1 March 2016; accepted 13 June 2016; published online 17 August 2016 
602

the transient model of Gong et al. ${ }^{17}$ to include skin surface lipids as a layer distinct from the underlying stratum corneum.

Very few physiologically based pharmacokinetic (PBPK) models have been developed for phthalates, and the ones that have been published were used to study disposition of phthalates in laboratory animals. ${ }^{19-21}$ The authors are not aware of the use of PBPK models to study human exposure to plasticizers. However, simple empirical pharmacokinetic (PK) models have been developed for humans and applied to general population exposure situations. $^{22-25}$ These models for di-2-ethylhexylphthalate (DEHP), DnBP, diisobutyl phthalate (DiBP), and Hexamoll DINCH were developed from human dosing experiments on one or more individuals who self-dosed at levels known to be substantially higher than background (but still known to be well below levels of health concern), and then metabolites were measured in blood for up to $6 \mathrm{~h}$ and urine samples for up to $48 \mathrm{~h}$ after dosing. The models were built around the data-they were toxicokinetic models whose purpose was to replicate the experimental data. As such, they had "blood" and "bladder" reservoirs that maintained the mass balance of phthalate metabolites and were able to predict the pattern of excretion of the metabolites seen in the experiments. Once calibrated, they can be used in general exposure scenarios where the exposure inputs are time and amount of phthalate assumed to be absorbed as parent into the blood reservoir in units of mass/time (or mass/body weight-time), and time and amount of urine voids. Of course, the assumption in these types of general model usages is that the calibrated parameters developed in the controlled human dosing experiments are suitable for general use.

In the present paper, a linked modeling approach was used to study the exposure of individuals to airborne vapor-phase DnBP. Chamber experiments with human subjects exposed to elevated levels of vapor-phase $\mathrm{DnBP}^{10}$ provided the data for this linked modeling study. These data included concentrations of DnBP in the chamber, and times and full urine volumes that were measured for DnBP metabolites starting from the volunteers' time in the chamber $(6 \mathrm{~h})$ and for 48 subsequent hours. The transient dermal model developed by Gong et al. ${ }^{17}$ was the initial candidate model selected to estimate transport of gas-phase DnBP from air through the skin to blood. Initial testing of the model of Gong et al. ${ }^{17}$ with these data sets led to the realization that the modeled absorption of DnBP was substantially more than could be accounted for by the measured excretion of the DnBP metabolites. This led to changes in the model, as described in Morrison et al. ${ }^{18}$ and summarized below. This adjusted model was linked to the toxicokinetic model developed by Lorber and $\mathrm{KoCh}^{25}$ to model excretion patterns of DnBP metabolites. In addition to modeling dermal uptake from air to blood, inhalation intake was also modeled, taking advantage of the fact that a portion of the data from these experiments was generated with the volunteers breathing the air that contained elevated levels of DnBP. Dynamic modeling of dermal uptake and inhalation intake provided a timevarying dose to blood that was input for the PK model; the PK model then predicted the delivery and excretion of DnBP metabolites for each of the urination events. A comparison of predicted and observed excretions of DnBP metabolites permitted an evaluation of the performance of the linked model approach. If the linked model is deemed successful, it can be used to enhance our understanding of potential exposures that are associated with DnBP, not only in situations with unusually high air concentrations but also under more typical indoor conditions. Such an exercise was undertaken to conclude the analysis. A typical indoor concentration of DnBP was determined from published measured values. The linked models routed inhalation and dermal permeation exposures to the "bladder" to predict daily mass excretions of a key DnBP metabolite. By dividing this mass by a typical daily urine volume, we were able to compare the resulting concentration with measured concentrations in a national survey. This permits a rough estimation of the relative contribution of inhalation and dermal uptake to total phthalate uptake from all pathways.

\section{METHODS}

\section{Chamber Experiments}

In 2014, chamber experiments were conducted to evaluate transdermal uptake of DnBP and DEP directly from air. ${ }^{10}$ These experiments took place at the Technical University of Denmark, and the protocol was approved by the Capital Region of Denmark Committee for Research Ethics. Participants provided informed consent before participation. The portion of the experiments entailing exposure to DnBP were used in the present modeling study. Six male volunteers, aged 27 to 66 years, were divided into groups 1 and 2. Each 3-member group was exposed to elevated concentrations of DnBP in a $55 \mathrm{~m}^{3}$ chamber for $6 \mathrm{~h}$. Group 1 comprised participants who are identified as P1, P2, and P3 in this paper, and group 2 includes P4, P5, and P6. On Tuesday of the first week, group 1 was exposed while wearing hoods and breathing air with background (low) concentrations of DnBP; hence, exposure was primarily via the dermal pathway. On Tuesday of the second week, group 1 was exposed without hoods and breathing chamber air; hence, exposure was via both the dermal and the inhalation pathway. The conditions were reversed for group 2, who were exposed without hoods on Wednesday of the first week and while wearing hoods on Wednesday of the second week. The concentrations of DnBP were measured seven times in the chamber air during each exposure period, resulting in the following average concentrations over $6 \mathrm{~h}$ in the chamber: group 1: hoods on $123 \mu \mathrm{g} / \mathrm{m}^{3}$ and hoods off $140 \mu \mathrm{g} / \mathrm{m}^{3}$; group 2: hoods on $140 \mu \mathrm{g} / \mathrm{m}^{3}$ and hoods off $114 \mu \mathrm{g} / \mathrm{m}^{3}$. During the chamber exposures the participants were clothed only in shorts and were seated most of the time. One or two background urine samples were taken before entering the chamber, and the participants provided one full volume urine sample during the chamber exposure. After leaving the chamber, full volumes of urine were collected for each urination over the next $48 \mathrm{~h}$. The urine was subsequently analyzed for DnBP metabolites, mono-n-butyl phthalate (MnBP), and 3-hydroxy-mono-n-butyl phthalate (3OH-MnBP). The amounts of MnBP and $3 \mathrm{OH}-\mathrm{MnPB}$ excreted at each urination provided the key dependent observations in the present study. The independent model inputs of inhalation intake and dermal uptake were based on the measured air concentrations and participant specific or literature derived parameters. Further details on the experimental design and results can be found in Weschler et al. ${ }^{10}$

\section{Inhalation Intake and Dermal Uptake}

Inhalation intakes for modeling were calculated as the product of individual-specific inhalation rates and the air concentration within the chamber. The inhalation rates specific to each of the 6 participants were developed in Weschler et al. ${ }^{10}$ (see Figure 3 ). They were developed for both DEP and DnBP results; the rates for each individual were similar between the two phthalates, although the inhalation rates between individuals were different. The average per individual (average of breathing rates between the two phthalates) used in this assessment were: $P 1=0.64 \mathrm{~m}^{3} / \mathrm{h}, \quad P 2=0.72 \mathrm{~m}^{3} / \mathrm{h}, \quad P 3=0.84 \mathrm{~m}^{3} / \mathrm{h}, \quad P 4=0.63 \mathrm{~m}^{3} / \mathrm{h}$, $P 5=0.45 \mathrm{~m}^{3} / \mathrm{h}$, and $\mathrm{P} 6=0.60 \mathrm{~m}^{3} / \mathrm{h}$. These are consistent with recommendations provided by the EPA Exposure Factors Handbook (EFH): ${ }^{26}$ for "sleep and nap" as well as "sedentary/passive" the rate is $0.26 \mathrm{~m}^{3} / \mathrm{h}$, for "light intensity" the rate is $0.72 \mathrm{~m}^{3} / \mathrm{h}$, and for "moderate intensity" the rate is $1.62 \mathrm{~m}^{3} / \mathrm{h}$. During exposures in which the participants did not wear a hood, the measured chamber concentrations were used to calculate the inhalation intakes. For example, for P1, the calculated inhalation intake for the "hood-off" condition was $89.6 \mu \mathrm{g} / \mathrm{h}$ (i.e., $140 \mu \mathrm{g} / \mathrm{m}^{3} \times 0.64 \mathrm{~m}^{3} / \mathrm{h}$ ). During exposures in which the participants wore a hood, inhalation intakes were calculated using the concentration of DnBP measured within the breathing hood (e.g., for P1 the calculation is $5.7 \mu \mathrm{g} / \mathrm{m}^{3} \times 0.64 \mathrm{~m}^{3}$ / $\mathrm{h}=3.6 \mu \mathrm{g} / \mathrm{h})$.

The procedure for modeling transdermal uptake was developed in Morrison et al. ${ }^{18}$ This model extended that of Gong et al. ${ }^{17}$ by including a layer of skin surface lipids and the influence of clothing. Briefly, dynamic phthalate uptake by dermal capillaries depends on transport by convective mass transfer through the air boundary layer adjacent to skin, accumulation in skin surface lipids, and Fickian diffusion through the stratum corneum and viable epidermis. After the $6 \mathrm{~h}$ bare-skin exposure period, participants don clothing; in the simulation this is modeled as a perfect 
sink located very close, but not touching, the skin. All input parameters are participant specific (air concentration and exposed skin area) and from literature or estimated independently (the convective mass-transfer coefficient, the thickness of each layer, air layer partition coefficients, diffusion coefficients). For the simulations here, we used the same set of parameters shown in Tables 1 and 2 (nominal parameters) of Morrison et al. ${ }^{18}$; many of these are listed in Supplementary Table S1. The dynamic dermal uptake of DnBP was generated for each participant for both hooded and non-hooded experiments. These results, unique to each participant and experiment, were then used as input to the pharmacokinetic model. Morrison et al. ${ }^{18}$ found that inclusion of clothing and skin surface lipids resulted in a significant improvement over previous models in predictions of overall dermal uptake for the participants described in Weschler et al. ${ }^{10}$ Predicted uptake of DEP was close to the mean value for all participants; however, predicted uptake of DnBP was 2.3 times greater than the measured values averaged over all participants. Inclusion of a one-time removal of skin surface lipids to clothing made a small improvement in the predictions for DEP and a larger improvement in the predictions for DnBP (see Figure 4 of Morrison et al. ${ }^{18}$ ). As the simulations applied in the current paper do not include removal of skin surface lipids, they are anticipated to result in an overprediction of transdermal uptake of DnBP that will range from a factor of 1.4 to 4.5 , depending on the individual simulated.

\section{PK Modeling}

The PK model for DnBP was described in Lorber and Koch. ${ }^{25}$ The model contains a "blood" and a "bladder" reservoir. Intakes are deposited directly into the "blood" reservoir as parent $\mathrm{DnBP}$, and within the blood reservoir, are metabolized to $\mathrm{MnBP}, 3 \mathrm{OH}-\mathrm{MnBP}, 4 \mathrm{OH}$-mono-n-butyl phthalate $(4 \mathrm{OH}-$ $\mathrm{MnBP}$ ), and 3carboxymono-propyl phthalate (MCPP). These metabolites are routed to the "bladder" reservoir, and the full amount in the reservoir at the time of an urination event is excreted. The model was calibrated to data from a single individual self-dosing with $5 \mathrm{mg}$ of labeled DnBP. Six measurements in blood up to $7 \mathrm{~h}$ after ingestion provided the data necessary to calibrate the metabolism algorithm in blood. Full urine volumes up to $48 \mathrm{~h}$ were obtained and also measured for DnBP metabolites, and these data provided the necessary information to calibrate the delivery of metabolites to the bladder reservoir. The toxicokinetics of DnBP metabolism as determined in a human experiment is as follows: $\sim 92 \%$ of a dose is excreted in urine as these metabolites within $24 \mathrm{~h}$, with MnBP explaining $\sim 84 \%$ of the $92 \%$, with the other metabolites noted explaining $\sim 8 \%{ }^{27}$ Further details of the model development can be found in Lorber and Koch. ${ }^{25}$

All toxicokinetic parameter values determined in Lorber and $\mathrm{Koch}^{25}$ were used without change in this application. Independent inputs to the model for the current study include the dermal uptake and inhalation intake, both of which were input into the blood reservoir as the starting point of the simulation. The dependent observations were the measured metabolites in urine samples, one taken while still in the chamber, and then every urination until $48 \mathrm{~h}$ after leaving the chamber for a total $54 \mathrm{~h}$ of simulation. These urine samples were measured only for two of the DnBP metabolites-MnBP and $3 \mathrm{OH}-\mathrm{MnBP}$ - and hence model validation only focuses on these two metabolites. The model was set up on an Excel spreadsheet and run in 5 min increments for $54 \mathrm{~h}$.

\section{Simulation Strategy}

Three sets of modeling exercises were undertaken:

Hood-on, dermal exposure. Generally, this simulation was a test of the transdermal uptake modeling scenario only. There was a relatively small inhalation intake due to the background concentration of DnBP in the hood air, $5.7 \mu \mathrm{g} / \mathrm{m}^{3}$, compared with $\sim 130 \mu \mathrm{g} / \mathrm{m}^{3}$ in chamber air. This small inhalation intake was added to the dermal dose directly into the blood reservoir during the simulation. Also input into the model were urination times and volumes of urine for the six volunteers. The model predicted mass and concentration of excreted metabolite to compare with the corresponding measured quantities. The contribution from inhalation intake was small compared with dermal uptake; inhalation intake was $\sim 5 \%$ of total intake.

Hood-off, dermal and inhalation exposure. Inhalation intake and dermal uptake of DnBP were added and input into the blood reservoir at $5 \mathrm{~min}$ increments. Within the blood reservoir, DnBP was metabolized, routed to the bladder, and excreted as per the input times and volumes of urine for each of the six volunteers.

Inhalation-only exposure. These "hood-off" experiments provide an opportunity to evaluate the inhalation component of the linked model, in the same way that the "hood-on" experiments provided a test of the dermal penetration model. As there was minimal inhalation uptake during these hood-on experiments, it can be simplistically assumed that all of the excretion of DnBP metabolites of the participants in these hood-on experiments was due to dermal penetration only. An "inhalation-only" scenario was crafted from the "hood-off" experiments. First, the inhalation exposure intake was simply calculated as in the hood-off simulations: an air concentration times an individual-specific inhalation rate. The next task was to "craft" a set of observed excretions that would be due only to the inhalation exposures. This was done as follows. First, rates of MnBP excretion between urination events were ascertained for each individual from the hood-on experiments. Then, these hood-on excretion rates were used to amend the hood-off excretions for each participant, so that a new set of "observed" excretions from the hood-off experiments can represent inhalation-only excretions. These are not "observed" excretions in the true sense of raw data from the experiment; they will be hereafter described as "crafted" excretions for this reason. This subtraction was not straightforward, however, because the chamber concentration for the two scenarios was somewhat different and urination times and volumes do not align for the two experiments. Therefore, it was necessary to convert observations from the hood-on experiments to time-varying excretion rates to use in the hood-off experiments for each participant and account for the difference in the times of urine events, and for the chamber concentrations. An example of this procedure for participant P1 is shown in Table 1 for the hood-on experiment. The normalized excretion rate, of MnBP only, for each urination interval is determined by dividing the excreted metabolite mass by the time interval since the last urination and then multiplying by the ratio of the hood-off and hood-on chamber concentrations (140/123). As shown in Table 2, these results are aligned with the excretion rates from the hood-off experiments and subtracted. If that subtraction resulted in a total of $<0$, then the excreted amount was set to 0 (see several such events in the last column of Table 2). As seen in Table 2, the total excretion from

Table 1. Calculation of the rate of excretion for Participant 1, P1, resulting from transdermal uptake of DnBP when wearing a hood (see text for more detail).

\begin{tabular}{lccc}
\hline $\begin{array}{l}\text { Interval in } \\
\text { hours }\end{array}$ & $\begin{array}{c}\text { Mass MnBP } \\
\text { excreted, } \mu g\end{array}$ & $\begin{array}{c}\text { Hourly rate of } \\
\text { excretion of } \\
\text { MnBP, } \mu g / h^{\text {b }}\end{array}$ & $\begin{array}{c}\text { Hourly rate of } \\
\text { MnBP excretion } \\
\text { normalized, } \mu g / h^{c}\end{array}$ \\
\hline $0-3.50$ & 12 & 3.4 & 3.9 \\
$3.50-7.17$ & 44 & 12.0 & 13.7 \\
$7.17-10.25$ & 49 & 15.7 & 17.9 \\
$10.25-11.25$ & 7 & 6.8 & 7.7 \\
$11.25-12.25$ & 5 & 5.6 & 6.4 \\
$12.25-17.50$ & 39 & 7.4 & 8.4 \\
$17.50-24.83$ & 64 & 8.7 & 9.9 \\
$24.83-29.50$ & 6 & 1.1 & 1.3 \\
$29.50-33.67$ & 10 & 2.6 & 3.0 \\
$33.67-37.50$ & 9 & 2.4 & 2.7 \\
$37.50-46.67$ & 19 & 2.0 & 2.3 \\
$46.67-50.83$ & 8 & 1.9 & 2.2 \\
$50.83-52.42$ & 1 & 0.5 & 0.6 \\
$52.42-55.50$ & 2 & 0.9 & 1.0 \\
\hline
\end{tabular}

${ }^{a}$ Hour " 0 " is when the participant enters the chamber. ${ }^{\text {b}}$ The hourly rate of excretion is calculated as $M(t 1) /(\mathrm{HR}(t 1)-\mathrm{HR}(t 0))$, where $M$ is the mass $(\mu \mathrm{g})$ of MnBP excreted, $H R(t 0)$ and $H R(t 1)$ are the times (h) for the previous event and the current event, respectively. For example, the rate between the first and second excretion is $(44) /(7.17-3.5)=12.0^{c}$ As the hourly rate of excretion, column 2, was based on "hood-on" experiments, this rate was normalized considering the difference in air concentration in the adjustment applied to the "hood-off" experiments. In this case, the "hood-on" concentration was $123 \mu \mathrm{g} / \mathrm{m}^{3}$, whereas for "hood off" the concentration was $140 \mu \mathrm{g} / \mathrm{m}^{3}$, necessitating an upward adjust of $140 / 123$ or 1.14 . 
Table 2. Calculation of the removal of excretion mass of MnBP from urine events of participant P1 that would be due to transdermal uptake in the hood-off experiment (see text for more detail).

\begin{tabular}{|c|c|c|c|c|}
\hline Hour ${ }^{\mathrm{a}}$ & $\begin{array}{l}\text { Total mass } \\
\text { MnBP } \\
\text { excreted, } \mu g^{b}\end{array}$ & $\begin{array}{c}\text { Rate of } \\
\text { excretion } \\
\text { applied, } \mu g / h^{c}\end{array}$ & $\begin{array}{l}\text { Number of } \\
\text { hours since } \\
\text { last excretion }\end{array}$ & $\begin{array}{l}\text { Mass excreted } \\
\text { due to inhalation } \\
\text { only, } \mu g^{\mathrm{e}}\end{array}$ \\
\hline 3.08 & 69 & 3.9 & 3.08 & 57.0 \\
\hline 5.25 & 116 & 13.7 & 2.17 & 86.3 \\
\hline 6.42 & 85 & 13.7 & 1.17 & 69.0 \\
\hline 8.33 & 93 & 17.9 & 1.91 & 58.8 \\
\hline 11.08 & 45 & 14.1 & 2.75 & 6.2 \\
\hline 12.42 & 16 & 7.7 & 1.34 & 5.7 \\
\hline 13.25 & 6 & 8.4 & 0.83 & 0.0 \\
\hline 13.58 & 5 & 8.4 & 0.33 & 2.2 \\
\hline 23.25 & 143 & 9.5 & 9.67 & 51.1 \\
\hline 26.00 & 23 & 6.8 & 2.75 & 4.3 \\
\hline 31.08 & 9 & 1.5 & 5.08 & 1.4 \\
\hline 33.33 & 5 & 3 & 2.25 & 0.0 \\
\hline 37.42 & 7 & 2.7 & 4.09 & 0.0 \\
\hline 41.33 & 5 & 2.3 & 3.88 & 0.0 \\
\hline 46.50 & 11 & 2.2 & 5.17 & 0.0 \\
\hline 49.42 & 7 & 2.2 & 2.92 & 0.6 \\
\hline 51.42 & 1 & 2.2 & 2.00 & 0.0 \\
\hline 54.33 & 32 & 0.7 & 2.91 & 30.0 \\
\hline Total & 678 & & & 372 \\
\hline \multicolumn{5}{|c|}{$\begin{array}{l}\text { aHour " } 0 \text { " is when the participant enters the chamber. }{ }^{\text {b}} \text { The observec } \\
\text { unadjusted data from the hood-off experiment for P1. "Approximatec } \\
\text { excretion rates from transdermal uptake determined from Table } 1 \text {. 'Hour o } \\
\text { the excretion minus the hour of the preceding excretion. "Original mas } \\
\text { excretion minus (hours since last excretion } \times \text { hourly excretion rate); if the } \\
\text { "corrected mass" is calculated to be }<0 \text {, it is set to zero. For example, for the } \\
\text { excretion at } 5.25 \mathrm{~h} \text {, the corrected mass is: } 116-(2.17 \times 13.7)=86.3 \mu \mathrm{g} \text {. }\end{array}$} \\
\hline
\end{tabular}

that experiment over $54 \mathrm{~h}$ (starting from when P1 entered the chamber at time 0 ) was $678 \mu \mathrm{g}$, and after subtraction for the dermal contribution, the total excretion was reduced to $372 \mu \mathrm{g}$. Therefore, this total of $372 \mu \mathrm{g}$ represents that portion of the total excretion due only to inhalation. This procedure was followed for all participants, P1 to P6.

Finally, it is noted that none of the simulations included background intakes of DnBP that the participants experienced once leaving the chamber and which undoubtedly influenced excretion of DnBP metabolites in the $48 \mathrm{~h}$ after leaving the chamber. The implications of this decision are discussed in the Results section below.

\section{RESULTS}

Figure 1 shows an example set of inputs, calculated from the dynamic model, ${ }^{18}$ to one of the linked model simulations. These modeled inputs include the rate of absorbed doses from inhalation and dermal uptake for participant P1 in the "hood off" experiments. The rate is expressed in units of $\mu \mathrm{g} / 5 \mathrm{~min}$ ( $5 \mathrm{~min}$ is the time step of the linked model). As seen, the modeled inhalation dose is constant over $6 \mathrm{~h}$ of the experiment and then stops upon leaving the chamber, while the modeled dermal dose continues and even peaks once outside the chamber. In this example, over $54 \mathrm{~h}$ modeled dermal uptake loads about twice as much DnBP into the blood $(1210 \mu \mathrm{g})$ as modeled inhalation intake $(588 \mu \mathrm{g})$.

Figures 2, 3, 4 show comparisons of predicted and observed metabolite ( $\mathrm{MnBP}$ and $3 \mathrm{OH}-\mathrm{MnBP}$ ) concentrations and cumulative mass excreted in urine for participant $\mathrm{P} 1$ for the three simulations: hood on, hood off, and inhalation only. The comparable figures for the other participants, P2 to P6, are shown in the Supplementary Materials. The general trends described here for $\mathrm{P} 1$ are the same for both metabolites and for all volunteers in the three sets of

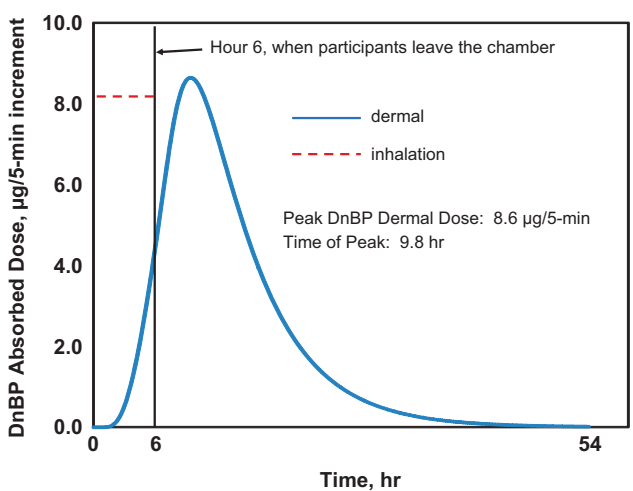

Figure 1. Model inputs of the rate of absorbed DnBP delivered to the blood (in units of $\mu \mathrm{g} / 5 \mathrm{~min}$ increment) from the $6 \mathrm{~h}$ chamber exposure for P1, "Hood Off", where the average air concentration was $140 \mu \mathrm{g} / \mathrm{m}^{3}$.

simulations. Five key trends are observed from the results shown in Figures 2, 3, 4:

(1) The timing of the peak urine metabolite concentrations and the rate of decline of concentrations to background levels after about the first 24-30 h (starting at time 0 when the participants enter the chamber) is well captured by the linked model.

(2) The relationship between $\mathrm{MnBP}$ and $3 \mathrm{OH}-\mathrm{MnBP}$ urine concentrations and mass excretions appear reasonably well captured by the linked model. Specifically, MnBP concentrations and cumulative excretions are more than an order of magnitude higher than those for $3 \mathrm{OH}-\mathrm{MnBP}$, and this is seen in both the data and the model predictions.

(3) Although the overall patterns of excretions of the two metabolites seem well predicted for P1, there is nonetheless an overprediction in concentrations and cumulative excretions of both metabolites over time. This overprediction is an anticipated contribution from the dermal uptake algorithm (see discussion in the Inhalation Intake and Dermal Uptake subsection in the Methods section above) and occurs from $\sim 10 \mathrm{~h}$ onwards (the participants leave the chamber at $6 \mathrm{~h}$ ). The same occurs with all participants in the simulations. As seen in Figure 1, because of storage in the stratum corneum the delivery of parent DnBP to blood continues throughout the $54 \mathrm{~h}$ of simulation, and peaks at $\sim 10 \mathrm{~h}$, and this is $4 \mathrm{~h}$ after leaving the chamber.

(4) For the crafted "inhalation-only" simulations, only MnBP was modeled, and the results are shown in Figure 4. The overall patterns in both concentrations and cumulative excretions appear well captured for participant P1. The predicted and observed concentrations are in fairly good agreement as are the cumulative excretions; the overprediction noted in observation 3 above is not seen for the crafted inhalation simulations.

(5) The participants in the hood-on and hood-off simulation sets, each of which included the dermal exposure pathway, appear to have been affected rather quickly by the elevated DnBP in the air. Specifically, the observed concentrations in the first and also the second urine event for these scenarios was higher than predicted by the model. This is seen in Figures 2 and 3 . However, the same is not true for the inhalation-only scenario in Figure 4, where dermal impacts are removed from the input stream and the excretions due to dermal uptake are also removed from the crafted excretions. In that case, there is a closer match between predicted and observed concentrations in the first two urine events. These trends are seen as well in 

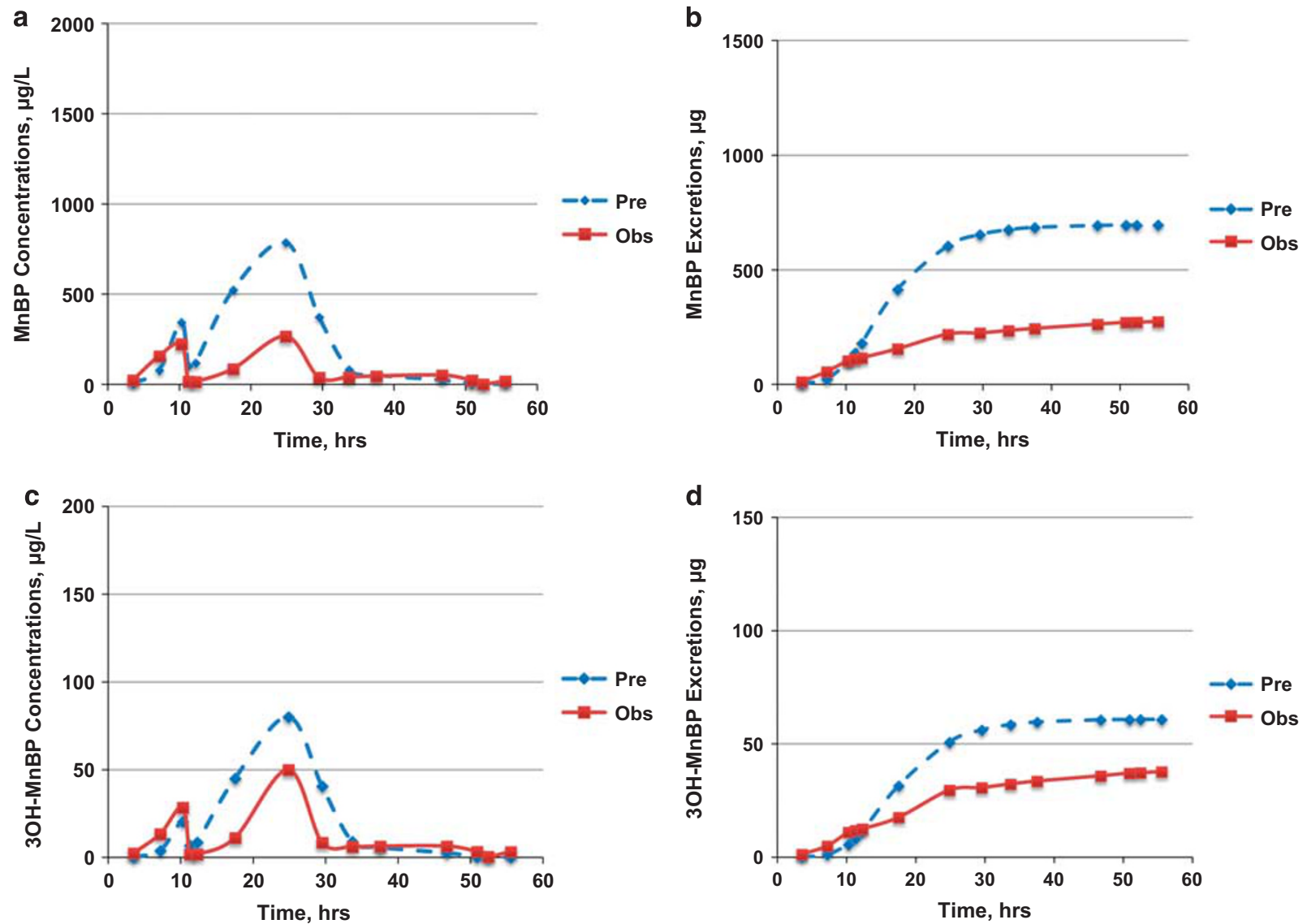

Figure 2. Comparison of predicted (blue lines with dots for predictions) and observed (red lines with dots for measurements) DnBP metabolite spot sample excretions for participant P1 in the "Hood-On" Simulation set. The four subgraphs are: (a) MnBP spot sample urine concentrations, (b) cumulative MnBP excretions, (c) $3 \mathrm{OH}-\mathrm{MnBP}$ spot sample urine concentrations, and (d) cumulative $3 \mathrm{OH}-\mathrm{MnBP}$ excretions.

other participants, as shown in the figures of the Supplementary Information. There appears an initial rush of airborne DnBP into and through the skin to quickly get into the body and be excreted; this is not captured by the dermal penetration model. The more immediate impact appears to be better captured for the inhalation pathway. Ultimately, fast initial dermal uptake has little effect on longer-term excretions, and real-world situations are likely to entail conditions more closely aligned to equilibrium and steady state compared with a sudden exposure to elevated airborne DnBP.

Table 3 shows the observed and predicted cumulative excretions over $54 \mathrm{~h}$ for all participants and the three simulation sets. The results of participant P1 in Table 3 are the final points in the cumulative graphs shown in Figures 2, 3, 4. Generally, observed cumulative excretions of MnBP in the hood-on experiments (set 1; avg $376 \mu \mathrm{g}$ ) are similar to the crafted excretions from inhalation-only (set 3 ; avg $311 \mu \mathrm{g}$ ). The cumulative observed excretion of MnBP with the hood-off experiments (set 2, both pathways) averages $663 \mu \mathrm{g}$, just about equal to the sum of hoodon and inhalation-only cumulative excretions. Even further, this sum also works out for each individual (not just the group average). For example, the hood-off cumulative excretion of P2 was $1020 \mu \mathrm{g}$, similar to the sum of the hood-on cumulative excretion and the inhalation-only cumulative excretion for P2, $983 \mu \mathrm{g}(590+393 \mu \mathrm{g})$. The individual with the lowest observed excretion for the hood-off experiment, P5 at $254 \mu \mathrm{g}$, had an observed sum of $295 \mu \mathrm{g}$. These equivalencies are not unexpected, and indeed they provide a secondary confirmation of the approach used to craft an inhalation-only set of individualspecific excretions; the sum of an inhalation-only excretion and a dermal-only excretion should equal the observed "total" excretion when both pathways are involved.

The anticipated overprediction because of the transdermal uptake algorithm is reflected in the modeled excretions. For the hood-on scenario, the model predicts an average cumulative excretion of $676 \mu \mathrm{g}$ that is nearly double the average of $376 \mu \mathrm{g}$ observed for that scenario. For the inhalation-only scenario, the model predicts an average cumulative excretion of $340 \mu \mathrm{g}$ that is very near the average crafted cumulative excretion of $311 \mu \mathrm{g}$. Indeed, the rank ordering of low to high excretions (from P5 lowest to P3 highest) is identical for both observed and predicted in this inhalation-only scenario. Similar to the hood-on scenario, the model overpredicts excretions for hood-off scenario, averaging $1018 \mu \mathrm{g}$ predicted compared with the average observed of $663 \mu \mathrm{g}$. Again, this difference is anticipated and due to the model overpredicting excretion when the dermal pathway is involved, as was discussed in the Methods section above.

This observation that the model appears to do well in characterizing inhalation intake and excretion but not as well in characterizing dermal uptake and excretion is consistent with the overpredictions of transdermal uptake of DnBP described in Morrison et al. ${ }^{18}$ They performed a sensitivity analysis on key parameters and suggested mechanisms that may reduce DnBP uptake; results are summarized in Figure 5 of Morrison et al. ${ }^{18}$ Transdermal uptake is especially sensitive to skin surface lipids because of the relatively large DnBP partition coefficient between 
a

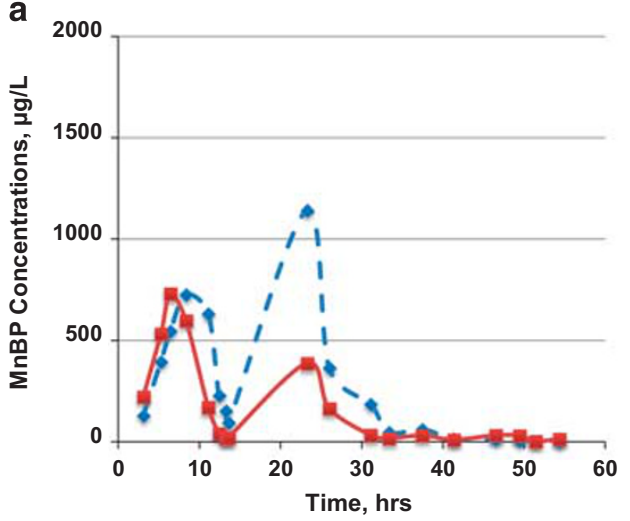

c

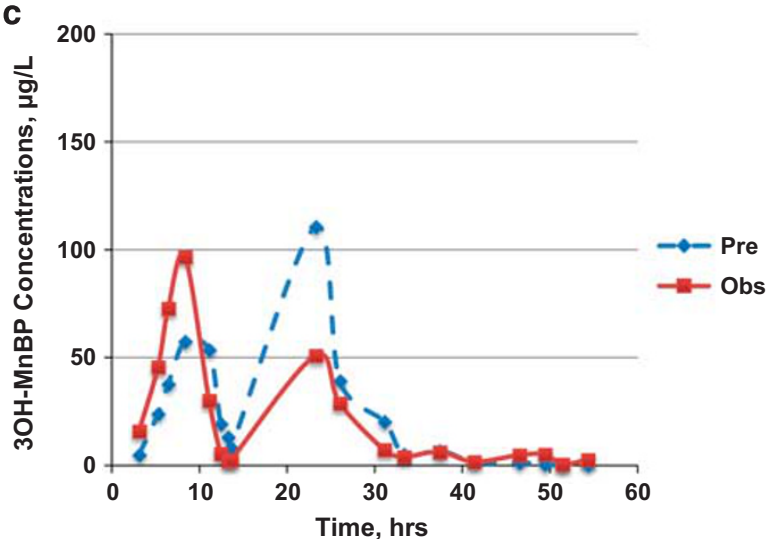

b

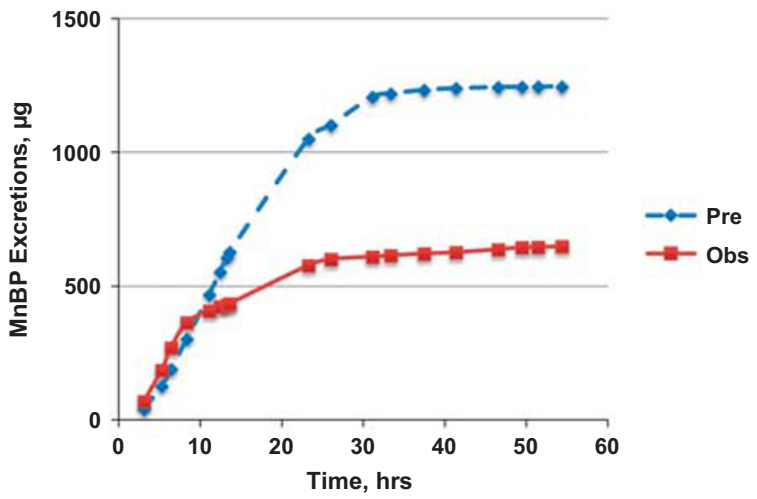

d

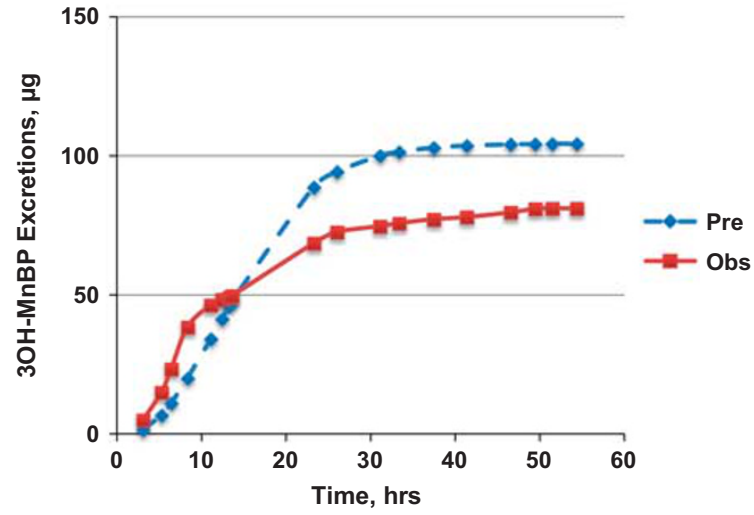

Figure 3. Comparison of predicted (blue lines with dots for predictions) and observed (red lines with dots for measurements) DnBP metabolite spot sample excretions for participant P1 in the "Hood-Off" Simulation set. The four subgraphs are: (a) MnBP spot sample urine concentrations, (b) cumulative MnBP excretions, (c) $30 \mathrm{H}-\mathrm{MnBP}$ spot sample urine concentrations, and (d) cumulative $30 \mathrm{H}-\mathrm{MnBP}$ excretions.
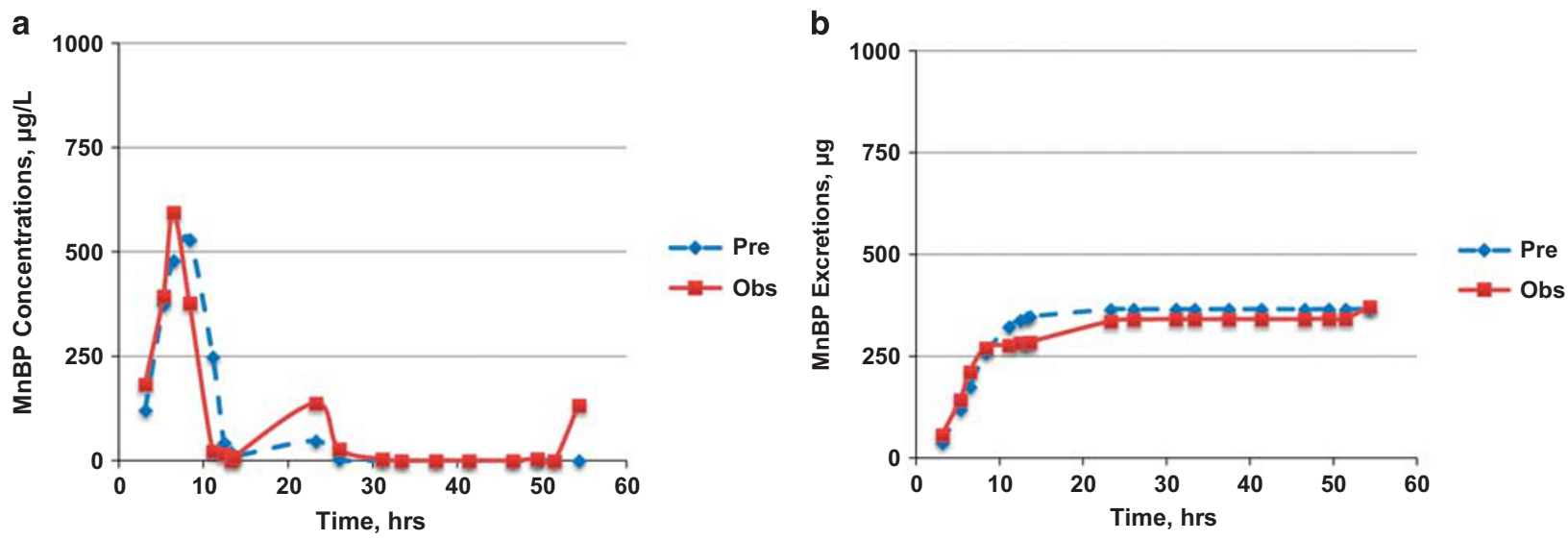

Figure 4. Comparison of predicted (blue lines with dots for predictions) and observed (red lines with dots for crafted measurements) MnBP spot sample excretions for participant P1 in the "Inhalation-Only" Simulation set. The two subgraphs are: (a) MnBP spot sample urine concentrations and (b) cumulative excretions of MnBP.

lipids and air. Certain partition coefficients used in the transdermal model-those between skin surface lipids and air $\left(K_{\text {ssl g }}\right)$ and between the stratum corneum and air $\left(K_{\text {sc }_{-} g}\right)$-are difficult to estimate for DnBP and may be off as much as an order of magnitude (a table of physical and chemical properties of DnBP is provided in Supplementary Materials). Removal of skin surface lipids by transfer to clothing or washing was also predicted to significantly reduce dermal uptake of DnBP (but not DEP). A single transfer of skin surface lipids to clothing after participants leave the exposure chamber was predicted to reduce DnBP uptake by $30 \%{ }^{18}$ Further removal by washing or transfer to new clothing or bedding would also reduce uptake. Subject-to-subject variability may also be influential.

Weschler et al. ${ }^{10}$ observed that normalized dermal uptake of both phthalates studied in these chamber experiments, DEP and $\mathrm{DnBP}$, increased with age, with the lowest amounts of excreted 
Table 3. Observed and predicted total excretions over $54 \mathrm{~h}(6 \mathrm{~h}$ in the chamber and then additional $48 \mathrm{~h}$ ) for the six participants, P1 to P6, for hood on, hood off, and inhalation exposure only.

\begin{tabular}{|c|c|c|c|c|}
\hline \multirow[t]{3}{*}{ Participant } & \multicolumn{4}{|c|}{ Cumulative excretions, $\mu g$} \\
\hline & \multicolumn{2}{|c|}{$M n B P$} & \multicolumn{2}{|c|}{$3 O H-M n B P$} \\
\hline & Observed & Predicted & Observed & Predicted \\
\hline \multicolumn{5}{|c|}{ (1) Hood-on experiments } \\
\hline P1 & 274 & 697 & 38 & 61 \\
\hline P2 & 590 & 682 & 57 & 60 \\
\hline P3 & 442 & 548 & 24 & 48 \\
\hline P4 & 307 & 696 & 22 & 61 \\
\hline P5 & 162 & 727 & 14 & 64 \\
\hline P6 & 484 & 705 & 31 & 62 \\
\hline \multicolumn{5}{|c|}{ (2) Hood-off experiments } \\
\hline P1 & 649 & 1189 & 81 & 104 \\
\hline P2 & 1020 & 1216 & 122 & 106 \\
\hline P3 & 906 & 1122 & 51 & 98 \\
\hline P4 & 529 & 881 & 32 & 77 \\
\hline P5 & 254 & 824 & 32 & 72 \\
\hline P6 & 589 & 874 & 62 & 77 \\
\hline \multicolumn{5}{|c|}{ (3) Inhalation-only simulations ${ }^{a}$} \\
\hline P1 & 342 & 366 & \multicolumn{2}{|c|}{$\begin{array}{c}\text { Not applicable; } \\
\text { inhalation simulations } \\
\text { focused only on MnBP }\end{array}$} \\
\hline P2 & 393 & 411 & & \\
\hline P3 & 471 & 480 & & \\
\hline P4 & 306 & 293 & & \\
\hline P5 & 133 & 209 & & \\
\hline P6 & 220 & 279 & & \\
\hline
\end{tabular}

(1) Hood on, where the exposure is dermal absorption only; (2) hood off, where the exposure is both inhalation and dermal absorption; and (3)

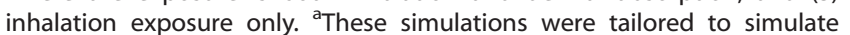
inhalation only starting from the Hood-Off data set. The input stream was altered to only include inhalation inputs and the "observed" excretions were renamed "crafted" to indicate that they were developed starting from the hood-off experiments and amended to remove that portion of the total excretion that was surmised to be due to dermal uptake. See text for more detail.

metabolites in the hood-on experiments seen in the youngest participant. (The ages of the participants were 27 (P5), 33 (P1), 34 (P4), 37 (P6), 47 (P2), and 66 (P3) years.) The agreement between modeled and measured results is best for the 66- and 47-year-old subjects and worst for the 27-year-old subject. However, the only person-specific factor considered in the dermal permeation modeling was exposed body surface area. Neither skin thickness nor skin hydration (which both decrease with age) were measured and could not be included in the dermal model. The sensitivity analysis in Morrison et al. ${ }^{18}$ suggested that normal variability in the thickness of the skin surface lipids and stratum corneum could result in uptake that varies by a factor of two or more. Diffusivity of DnBP in the stratum corneum is also uncertain and given its high $K_{\mathrm{ow}}$ and molecular weight, it is very difficult to measure for the isolated stratum corneum. Instead, it was calculated using the methodology in Gong et al. ${ }^{17}$ for partially hydrated stratum corneum but was not varied independently. Instead, it was varied with the reciprocal of the partition coefficient (consistent with Gong et al. ${ }^{17}$ ); predicted uptake was weakly sensitive to this parameter.

The metabolic conversion model for DnBP was based on an oral dose of DnBP, and was calibrated based on the internal metabolism from a single individual (see Lorber and $\mathrm{KoCh}^{25}$ ) without any changes made for the different individuals of the experiment. This appeared to work well for inhalation. However, metabolism may differ in some respects for dermal uptake, as in the latter case DnBP bypasses enzymes in the stomach and intestines. Therefore, there may be a need to modify metabolic parameters in the PK model when applied to the dermal pathway. Finally, it was noted in the Methods section that background exposures to DnBP once leaving the chamber were not considered in the modeling but undoubtedly influenced excretion of metabolites in the $48 \mathrm{~h}$ after leaving the chamber. Had background exposures been considered, there would have been higher predicted excretions of $\mathrm{MnBP}$ and $\mathrm{3OH}-\mathrm{MnBP}$, and a larger gap between predicted and observed excretions of metabolites. However, we conclude that the impact of not considering background exposures would be small. Weschler et al. ${ }^{10}$ calculated the possible background-related excretions of $\mathrm{MnBP}$ and $3 \mathrm{OH}-\mathrm{MnBP}$ for each participant for the time outside the chamber during 6-54 h based on observed MnBP and $3 \mathrm{OH}-\mathrm{MnPB}$ excretions in the prechamber urination events and the last urination event near $54 \mathrm{~h}$. Based on their calculations, the average excretion of $\mathrm{MnBP}$ and $3 \mathrm{OH}-\mathrm{MnBP}$ over all participants for 7-54 h due only to background sources would be 68 and $6.1 \mu \mathrm{g}$, respectively. To put this in perspective, the total observed excretion of the metabolites including one urination event within the chamber and then all events outside the chamber averaged $377 \mu \mathrm{g} \mathrm{MnBP}$ and $31 \mu \mathrm{g} \mathrm{3OH-MnBP}$ for hood-on experiments, and $658 \mu \mathrm{g} \mathrm{MnBP}$ and $63 \mu \mathrm{g} \mathrm{3OH}-\mathrm{MnBP}$ for hood-off experiments. On average, only $\sim 18 \%$ of MnBP excretions for the hood-on and $10 \%$ of excretions for the hood-off experiments may have been due to background exposures once outside the chamber.

\section{DISCUSSION}

This study linked models of DnBP transdermal uptake and inhalation intake to a simple PK model to predict excretions of DnBP metabolites in urine over the $54 \mathrm{~h}$ period. The observed data were from a chamber experiment described in Weschler et al., ${ }^{10}$ where six mostly bare-skinned (shorts only) male adults were exposed to elevated levels of DnBP in air over a $6 \mathrm{~h}$ period, and full volumes of urine were obtained from the time the participants entered the chamber until $54 \mathrm{~h}$ later.

A key assumption in the modeling was that the chamber air concentrations were not limited in any way by inhalation and dermal uptake of the three individuals within the chamber for $6 \mathrm{~h}$. A simple mass balance was conducted that showed that removal by the individuals through modeled inhalation and dermal uptake was not limiting (calculation not shown). Indeed, consecutive hourly samples of air while the subjects were in the chamber showed consistent air concentrations. Therefore, we concluded that possible removal by exposure of the individuals in the chamber would not limit the amount available for any of them over time. The concentrations used in modeling were the average of the multiple samples collected during the exposure period.

The linked model generally captured the patterns of exposure and internal metabolism with regard to the timing of peak concentration, rapid decline to background concentrations, and the relationship between the two key metabolites of DnBP that were modeled (MnBP and $3 \mathrm{OH}-\mathrm{MnBP}$ ). However, there is a pattern of overprediction associated with dermal uptake that was anticipated based on the results of Morrison et al. ${ }^{18}$ We have compared observations and model predictions using results for a single representative participant (Figures 1, 2, 3, 4), for all participants (Table 3), and average results (text). The graphical patterns shown in Figures 2, 3, 4 for participant P1 are shown for all participants in the Supplementary Materials. However, we have not applied any statistical goodness-of-fit tests between model predictions and observations. Such tests would be fated to show poor results where dermal uptake was modeled because of this pattern of overprediction. 
There are two consistent patterns of differences between observations and modeled results associated with the dermal uptake pathway. In the first pattern, metabolites associated with transdermal uptake appear in urine much quicker than is modeled by the transdermal/toxicokinetic model. This was seen in higher observed concentrations in the first urine event while the participants were in the chamber, and to a lesser extent in the second urine event shortly after participants left the chamber. This pattern was not seen in the crafted inhalation-only scenarios. We are unclear as to the cause of this observation and did not pursue understanding or modeling it further. A second pattern that was observed was that the model then continues to overpredict excretions starting at approximately the $10 \mathrm{~h}$ mark, $4 \mathrm{~h}$ after participants leave the chamber. Ultimately, after $54 \mathrm{~h}$, the model has predicted between 1.1 and 4.5 times the observed cumulative excretion of $\mathrm{MnBP}$ and $3 \mathrm{OH}-\mathrm{MnBP}$ when transdermal uptake is involved, the hood-on and hood-off scenarios (see Table 3).

Despite a tendency for the present linked model to overpredict DnBP metabolite excretions, we are not disappointed with the results. Certainly, better fits could be obtained with different plausible model parameters. As discussed, model sensitivity exercises in Morrison et al. ${ }^{18}$ showed that a one-time removal of skin surface lipids, as might occur with donning of clothing for example, resulted in reduced dermal penetration of DnBP. We did not include any removal events that might include bathing in addition to donning and removing clothing. However, our intent was to assign model parameters using the best available information, which may have been previous calibrations (as in the toxicokinetic model), or using best literature values. Generally, we conclude that this exercise has provided a positive proof of concept of linking two dose models (transdermal and inhalation) to an internal kinetic model to characterize exposure to airborne DnBP.

This linked model gives us a tool to estimate the contribution that airborne DnBP can make to overall DnBP exposure in different settings. We demonstrate the linked model's capabilities with a simple characterization of general population exposure to airborne DnBP within the United States using the following approach. First, a typical background air concentration of DnBP was estimated based on measured levels reported in the literature. Then, the intake models were run to steady state to determine daily dermal and inhalation intakes. Intakes were run through the toxicokinetic model as was done for the chamber experiments, and daily steady-state mass of MnBP that gets delivered to the model's "bladder" (awaiting excretion) were determined. An amount of MnBP delivered over $24 \mathrm{~h}$ was divided by an average daily urine volume to get a representative concentration of MnBP. Finally, this was compared with representative urine concentrations of MnBP for US adults as measured in recent cycles of NHANES (National Health and Nutritional Evaluation Survey from $\left(\mathrm{CDC}^{4}\right)$. We emphasize that this is a simple demonstration of what can be accomplished with the linked model. There are a wide range of possible input variables and parameters, as will be described, and this should not be construed as a definitive attempt to characterize inhalation and dermal exposures to DnBP in the general US population.

The median airborne DnBP concentration reported by Rudel et al. $^{3}$ for 120 Cape Cod homes was $0.22 \mu \mathrm{g} / \mathrm{m}^{3}$. The US EPA CTEPP (Children's Total Exposure to Persistent Pesticides and Other Persistent Organic Pollutants) study reported median airborne concentrations of $0.23 \mu \mathrm{g} / \mathrm{m}^{3}$ in 129 North Carolina homes, $0.25 \mu \mathrm{g} / \mathrm{m}^{3}$ in 127 Ohio homes, $0.38 \mu \mathrm{g} / \mathrm{m}^{3}$ in 13 North Carolina daycare facilities, and $0.32 \mu \mathrm{g} / \mathrm{m}^{3}$ in 16 Ohio day-care facilities. ${ }^{28}$ Gaspar et al. ${ }^{2}$ report a median air concentration of $0.52 \mu \mathrm{g} / \mathrm{m}^{3}$ for 40 California day-care facilities. Tran and Kannan ${ }^{29}$ collected 60 indoor air samples from homes $(n=20)$, offices $(n=7)$, laboratories $(n=13)$, schools $(n=6)$, salons $(n=6)$, and public places $(n=8)$ in Albany, NY, and found a median of $0.06 \mu \mathrm{g} / \mathrm{m}^{3}$ over all settings; the highest concentrations were in salons with a median of $0.31 \mu \mathrm{g} / \mathrm{m}^{3}$. Based on these studies, we selected a representative air concentration of $0.2 \mu \mathrm{g} / \mathrm{m}^{3}$. Furthermore, we assume that this total concentration is all in the gas phase (at typical concentrations of indoor airborne particles, 95\% of DnBP is in the gas phase). ${ }^{30}$ Daily inhalation exposures were calculated assuming an hourly inhalation rate of $0.7 \mathrm{~m}^{3} / \mathrm{h}$, similar to the "light intensity" rate of inhalation of $0.72 \mathrm{~m}^{3} / \mathrm{h}$ recommended in the EPA EFH. ${ }^{26}$ Under these conditions, the total daily intake by inhalation is calculated as $0.042 \mu \mathrm{g} / \mathrm{kg}$ per day $\left(\left[0.7 \mathrm{~m}^{3} / \mathrm{h} \times 24 \mathrm{~h} /\right.\right.$ day $\times 0.2 \mu \mathrm{g} /$ $\left.\mathrm{m}^{3}\right) / 80 \mathrm{~kg}$, where $80 \mathrm{~kg}$ is the recommended adult body weight in $E^{2} A^{26}$ ). We ran the transdermal model to steady state with all key parameters unchanged from the chamber experiments, with the exception of the exposed body surface area that we assumed was $1.0 \mathrm{~m}^{2}$. The total body surface area for adults is $\sim 2 \mathrm{~m}^{2}$, with the head, arms, and hands (typical body parts assumed to be uncovered in dermal exposure scenarios) about one-quarter of that. The exposed area of $1.0 \mathrm{~m}^{2}$ assumes slight attenuation by clothing. Clothing can either decrease or amplify dermal uptake, depending on its history before it is worn. ${ }^{31}$ With these assumptions, the transdermal model was run to steady state, resulting in an estimated daily transdermal uptake of DnBP of $0.14 \mu \mathrm{g} / \mathrm{kg}$ per day. Note that for this simple example, transdermal uptake is $\sim 3$ times higher than inhalation intake. This is not the same as the result for the $6 \mathrm{~h}$ chamber experiments that showed comparable intakes for the two pathways. However, $6 \mathrm{~h}$ is too short a time for dermal uptake to approach steady state. At steady state (and with all model parameters as assigned), the model indicates that transdermal uptake exceeds inhalation by about a factor of three.

The linked models were run with these steady inputs from inhalation intake and dermal uptake for 4 days; the mass of MnBP excreted on the fourth day (steady state attained) was $9.9 \mu \mathrm{g}$, or on a body weight basis, $0.124 \mu \mathrm{g} / \mathrm{kg}$ per day. If we divide this excretion of $9.9 \mu \mathrm{g}$ by an average adult urine volume of $1.5 \mathrm{I}^{32}$ (comparable to "reference values" of $1.6 \mathrm{I}$ for males and $1.2 \mathrm{I}$ for females in $\mathrm{ICRP}^{33}$ ), we calculate an MnBP urine concentration of $6.6 \mu \mathrm{g} / \mathrm{l}$. The geometric mean concentrations of MnBP found in NHANES surveys 2005/6, 2007/8, and 2009/10 were 19.6, 18.9 and $14.6 \mu \mathrm{g} / \mathrm{l}$, respectively. ${ }^{4}$ It is noted that the dermal uptake model was found to overpredict penetration of DnBP. Uptake is sensitive to the removal of DnBP by mechanisms not included in this modeling exercise such as skin surface lipid transfer to clothing and removal by bathing. ${ }^{18}$ Hence, the prediction in this simple demonstration was more likely higher than would be predicted if such processes had been included. In addition, key inputs such as the typical background air concentration and the exposed body surface area were assigned values that may not accurately characterize the general US population. Finally, type of clothing worn and laundering practices can influence uptake of DnBP via the dermal pathway. Still, assuming our assignments are reasonable for purposes of demonstration, a prediction suggesting that airborne concentrations of DnBP could explain upwards of $25 \%$ of total exposures is fairly remarkable. Without being too precise, given all the assumptions and uncertainties of this simple exercise, we conclude that the combined pathways of inhalation and dermal uptake of airborne DnBP contribute a substantial fraction of exposure to DnBP in the general US population.

The chamber experiments described in Weschler et al. ${ }^{10}$ demonstrated the importance of the dermal pathway for exposure to DnBP and DEP. The linked models of this paper show the feasibility of additionally studying this pathway through the use of such models, and the application of the linked models in this example of general population exposure demonstrates their utility. Further exercises could be undertaken for DnBP exposures in different settings, and similar linked modeling could be done in the case of DEP, the other phthalate studied in the chamber experiments. 


\section{CONFLICT OF INTEREST}

The findings and conclusions in this report are those of the authors and do not necessarily represent the official position of the US Environmental Protection Agency.

\section{REFERENCES}

1 Fromme H, Lahrz T, Piloty M, Gebhart H, Oddoy A, Ruden H. Occurrence of phthalates and musk fragrances in indoor air and dust from apartments and kindergartens in Berlin (Germany). Indoor Air 2004; 14: 188-195.

2 Gaspar FW, Castorina R, Maddalena RL, Nishioka MG, McKone TE, Bradman A. Phthalate exposure and risk assessment in California child care facilities. Environ Sci Technol 2014; 48: 7593-7601.

3 Rudel RA, Camann DE, Spengler JD, Korn LR, Brody JG. Phthalates, alkylphenols, pesticides, polybrominated diphenyl ethers and other endocrine disrupting compounds in indoor air and dust. Environ Sci Technol 2003; 37: 4543-4553.

4 CDC (Centers for Disease Control and Prevention). 2015 Fourth National Report on Human Exposure to Environmental Chemicals, Updated Tables, February 2015. Atlanta, GA: Centers for Disease Control and Prevention. Available at http://www.cdc. gov/biomonitoring/pdf/FourthReport_UpdatedTables_Feb2015.pdf [accessed 12 May 2016].

5 Bekö G, Weschler CJ, Langer S, Callesen M, Toftum J, Clausen G. Children's phthalate intakes and resultant cumulative exposures estimated from urine compared with estimates from dust ingestion, inhalation and dermal absorption in their homes and daycare centers. PLoS One 2013; 8: e62442.

6 Wittassek M, Koch HM, Angerer J, Bruening T. Assessing exposure to phthalates the human biomonitoring approach. Mol Nutr Food Res 2011; 55: 7-31.

7 Wormuth $M$, Scheringer $M$, Vollenweider $M$, Hungerbuhler $K$. What are the sources of exposure to eight frequently used phthalic acid esters in Europeans? Risk Anal 2006; 26: 803-824.

8 Clark KE, David RM, Guinn R, Kramarz KW, Lampi MA, Staples CA. Modeling human exposure to phthalate esters: a comparison of indirect and biomonitoring estimation methods. Hum Ecol Risk Assess 2011; 17: 923-965.

9 Langer S, Weschler CJ, Fischer A, Beko G, Toftum J, Clausen G. Phthalate and PAH concentrations in dust collected from Danish homes and daycare centers. Atmos Environ 2010; 44: 2294-2301.

10 Weschler CJ, Bekö G, Koch HM, Salthammer T, Schripp T, Toftum J et al. Transdermal uptake of diethyl phthalate and di(-butyl) phthalate directly from air: Experimental verification. Environ Health Perspect 2015; 123: 928-934.

11 Piotrowski JK. Evaluation of exposure to phenol: absorption of phenol vapour in the lungs and through the skin and excretion of phenol in urine. $\mathrm{Br} J$ Ind Med 1971; 28: 172-178.

12 Piotrowski J. Further investigations on the evaluation of exposure to nitrobenzene. Br J Ind Med 1967; 24: 60-65.

13 Weschler CJ, Nazaroff WW. Dermal uptake of organic vapors commonly found in indoor air. Environ Sci Technol 2014; 48: 1230-1237.

14 Weschler CJ, Nazaroff WW. Semivolatile organic compounds in indoor environments. Atmos Environ 2008; 42: 9018-9040.

15 Weschler CJ, Nazaroff WW. SVOC exposure indoors: fresh look at dermal pathways. Indoor Air 2012; 22: 356-377.

16 Mitragotri S. A theoretical analysis of permeation of small hydrophobic solutes across the stratum corneum based on Scaled Particle Theory. J Phar Sci 2002; 91: 744-752.

17 Gong M, Zhang Y, Weschler CJ. Predicting dermal absorption of gas-phase chemicals: transient model development, evaluation, and application. Indoor Air 2014; 24: 292-306.

18 Morrison G, Weschler CJ, Bekö G. Dermal uptake directly from air under transient conditions: Advances in modeling and comparisons with experimental results for human subjects. Indoor Air 2016 (doi: 10.1111/ina.12277; http://onlinelibrary.wiley. com/journal/10.1111/(ISSN)1600-0668/earlyview).
19 Keys DA, Wallace DG, Kepler TB, Conolly RB. Quantitative evaluation of alternative mechanisms of blood and testes disposition of di(2-ethylhexyl) phthalate and mono(2-ethylhexyl) phthalate in rats. Tox Sci 1999; 49: 172-185.

20 Clewell RA, Kremer JJ, Williams CC, Campbell JL, Andersen ME, Borghoff SJ. Tissue exposures to free and glucuronidated monobutylyphthalate in the pregnant and fetal rat following exposure to di-n-butylphthalate: evaluation with a PBPK model. Tox Sci 2008; 103: 241-259.

21 Karara AH, Hayton WL. Pharmacokinetic model for the uptake and disposition of di-2-ethylhexyl phthalate in sheepshead minnow Cyprinodon variegatus. Aquatic Tox 1984; 5: 181-195.

22 Schutze A, Lorber M, Gawrych K, Kolossa-Gehring M, Apel P, Bruning T et al. Development of a multi-compartment pharmacokinetic model to characterize the exposure to Hexamoll ${ }^{\circ}$ DINCH $^{\circ}$. Chemosphere 2015; 128: 216-224.

23 Lorber M, Angerer J, Koch HM. A simple pharmacokinetic model to characterize exposure of Americans to Di-2-ethylhexyl phthalate. J Expo Sci Environ Epidemiol 2010; 20: 38-53.

24 Lorber M, Calafat A. Dose reconstruction of di(2-ethylhexyl) phthalate using a simple pharmacokinetic model. Env Health Persp 2013; 20: 1705-1710.

25 Lorber M, Koch H. Development and application of simple pharmacokinetic models to study human exposure to di-n-butyl phthalate (DnBP) and diisobutyl phthalate (DiBP). Env Int 2013; 59: 469-477.

26 U.S. EPA. Exposure Factors Handbook 2011 Edition (Final). U.S. Environmental Protection Agency, Washington, DC, EPA/600/R-09/052F, 2011. Available at https:// cfpub.epa.gov/ncea/risk/recordisplay.cfm?deid = 236252 [accessed 12 May 2016].

27 Koch HM, Christensen KLY, Harth V, Lorber M, Bruning T. Di- $n$-butyl phthalate (DnBP) and diisobutyl phthalate (DiBP) metabolism in a human volunteer after single oral doses. Arch Toxicol 2012; 86: 1829-1839.

28 Morgan MK, Sheldon LS, Croghan CW, Chuang JC, Lordo RA, Wilson NK et al. A pilot study of children's total exposure to persistent pesticides and other persistent organic pollutants (CTEPP). Contract Number 68-D-99-011, Task Order 0002. US EPA National Exposure Research Laboratory: Research Triangle Park, NC 2004.

29 Tran TM, Kannan K. Occurrence of phthalate diesters in particulate and vapor phases in indoor air and implications for human exposure in Albany, New York, USA. Arch Environ Contam Toxicol 2015; 68: 489-499.

30 Weschler CJ, Nazaroff WW. SVOC partitioning between gas phase and settled dust indoors. Atmos Environ 2010; 44: 3609-3620.

31 Morrison GC, Weschler CJ, Bekö G, Koch HM, Salthammer T, Schripp T et al. Role of clothing in both accelerating and impeding dermal absorption of airborne SVOCs. $J$ Expo Sci Environ Epidemiol 2016; 26: 113-118.

32 Boron WF, Boulpaep EL. Medical Physiology: A Cellular and Molecular Approach Elsevier/Saunders: Philadelphia, PA, USA, 2005.

33 ICRP. Basic Anatomical and Physiological Data for Use in Radiological Protection Reference Values. ICRP Publication 89. Ann. ICRP 2002; 32 (3-4). Available at http:// www.icrp.org/publication.asp?id=ICRP\%20Publication\%2089.

(c) $\Theta$ This work is licensed under a Creative Commons AttributionBY NG ND NonCommercial-NoDerivs 4.0 International License. The images or other third party material in this article are included in the article's Creative Commons license, unless indicated otherwise in the credit line; if the material is not included under the Creative Commons license, users will need to obtain permission from the license holder to reproduce the material. To view a copy of this license, visit http:// creativecommons.org/licenses/by-nc-nd/4.0/

(c) The Author(s) 2017

Supplementary Information accompanies the paper on the Journal of Exposure Science and Environmental Epidemiology website (http:// www.nature.com/jes) 